\title{
Opciones de creatividad durante la práctica de actividades físicas de expresión y deportes artísticos desde la Spórtica
}

\author{
Juan Pedro Ribas ${ }^{1}$ \\ Mercè Mateu-Serra ${ }^{2}$
}

\section{RESUMEN}

Objetivo: Determinar las opciones de creatividad durante la práctica de las actividades físicas de expresión y de los deportes artísticos desde la Spórtica. Introducción: La Praxiología motriz sugiere que las opciones creativas de las actividades físicas de expresión y los deportes artísticos deberían formar parte de la Educación física. ¿Cuáles son las diferencias entre los procesos creativos que surgen durante la práctica? La Spórtica trata de responder esta pregunta utilizando tres criterios: tipo de objetivos motores, tipo de objetivos externos y tipo de incertidumbre. Discusión: Los objetivos de las actividades de expresión y los deportes artísticos son "intentar obtener formas motrices asociadas a referencias estéticas/temáticas". Imitar, improvisar, variar y componer son cuatro procesos creativos. Los cuatro procesos utilizan los objetivos de las actividades de forma diferente. El grado de creatividad de los cuatro procesos depende del grado de reglamentación de la actividad y de la incertidumbre provocada en los participantes. Conclusión: De acuerdo a la Spórtica, el profesorado de Educación física puede elegir cuatro procesos creativos expresivos que conllevan tres grados de creatividad.

Palabras clave: Educación física. Creatividad. Expresión. Deporte artístico. Spórtica.

\footnotetext{
1 Universidad de Málaga, Málaga, Andalucía, Espanha.

2 Institut Nacional d'Educació Física de Catalunya, Barcelona, Catalunya, Espanha
}

\section{Correspondência:}

Juan Pedro Ribas. Departamento Didáctica de las lenguas, las Artes y el Deporte, Universidad de Málaga, Bulevar Louis Pasteur, 25, CEP 29010, Málaga España, Email: juanperibas2@gmail.com 


\section{Opções de criatividade durante a prática de atividades físicas de expressão e esportes artísticos a partir da Spórtica}

\section{RESUMO}

Objetivo: Determinar as opções de criatividade durante a prática de atividades físicas de expressão e esportes artísticos a partir da Spórtica. Introdução: A Praxiologia Motriz sugere que as opções criativas de atividades físicas de expressão e esportes artísticos devem fazer parte da Educação Física. Quais são as diferenças entre os processos criativos que surgem durante a prática? A Spórtica trata de responder a essa pergunta usando três critérios: tipo de objetivos motores, tipo de objetivos externos e tipo de incerteza. Discussão: Os objetivos das atividades de expressão e dos esportes artísticos são "tentar obter formas motrizes associadas às referências estéticas/temáticas". Imitar, improvisar, variar e compor são quatro processos criativos. Os quatro processos usam os objetivos das atividades de maneiras diferentes. O grau de criatividade dos quatro processos depende do grau de regulação da atividade e da incerteza causada nos participantes. Conclusão: Segundo a Spórtica, os professores de Educação Física podem escolher quatro processos criativos expressivos que envolvem três graus de criatividade.

Palavras-chave: Educação Física. Criatividade. Expressão. Esporte artístico. Spórtica.

\section{Creativity options during the practice of expressive physical activities and artistic sports from Sportics}

\section{ABSTRACT}

Objective: Establish the creativity options during the practice of expressive physical activities and artistic sports from Sportics. Introduction: Motor Praxeology suggests that the creative options of physical expressive activities and artistic sports should be part of PE. What are the differences between the creative processes that emerge during practice? Sportics tries to answer this question using three criteria: type of motor objectives, type of external objectives and type of uncertainty. Discussion: The aims of expressive activities and artistic sports are "trying to obtain motor shapes associated to aesthetic/thematic references". Imitating, improvising, varying and composing are four creative processes. The four processes use the activities objectives differently. The creativity degree of the four processes depends on the activity regulation degree and the uncertainty caused in the participants. Conclusion: According to Spórtica, PE teachers can choose four expressive creative processes that involve three creativity degrees.

Keywords: Physical Education. Creativity. Expression. Artistic sport. Sportics. 


\section{INTRODUCCIÓN}

Danza, actividades de circo, torres humanas, canta-juegos, representación de roles sociales, sombras chinas, baile deportivo, half-pipe, saltos acrobáticos, bmx, gimnasia artística, natación artística, patinaje artístico, exhibición de artes marciales, vuelo acrobático de cometas, taichí deportivo, concurso de mates, figuras con el yo-yo, pressing-catch y jugar a las casitas son ejemplos de actividades físicas de expresión.

Parlebas (2001 y 2017) ha resaltado el potencial de la práctica de actividades de expresión para fomentar la creatividad del alumnado. ¿Nos está invitando a la búsqueda de propuestas aplicables a la Educación física a partir de la Praxiología motriz?

Troya (2018, p. 34) plantea que la Praxiología motriz analice las actividades físicas de expresión y sus diferentes opciones creativas; solo así las actividades de expresión podrán formar parte de los contenidos de la Educación física: "es esencial que las clasificaciones de carácter específico abarquen todo el potencial que posee la conducta motriz, sin despojar a la educación motriz de su parte expresiva, rítmica, creativa y artística".

Respecto a las "clasificaciones específicas" procedentes de la Praxiología motriz, Troya (2018) concretamente menciona a los objetivos motores. El objetivo motor (HERNÁNDEZ MORENO et al., 2000, p. 97) es "el aspecto de la lógica interna que implica la demanda motriz prioritaria a conseguir por el/los participante/s y que da sentido a dicha práctica".

Para Ribas (2018) los análisis de las actividades físicas y deportes efectivamente parten de los objetivos motores, pero para entender el "sentido" de la práctica en ocasiones se requieren otros criterios asociados a los objetivos motores: los objetivos externos y la incertidumbre que tienen los participantes durante la práctica.

Según Hernández Moreno (Dir.) (2000) cuando los participantes tratan de alcanzar el objetivo motor (en el momento de la práctica de actividades físicas) se genera una dinámica, y que Ribas (2018) denomina "dinámica de la práctica", siendo la dinámica de la práctica el objeto de estudio de la Spórtica (Sportics, Sportique).

El objetivo del artículo se centra en determinar las opciones de creatividad durante la dinámica de la práctica de las actividades físicas de expresión y los deportes artísticos desde la Spórtica.

Las cuestiones que trataremos de responder sucesivamente en este 
artículo desde la Spórtica son las siguientes:

¿Cuáles son los objetivos motores que identifican a las actividades físicas de expresión y a los deportes artísticos durante la dinámica de la práctica?

¿Son suficientes los objetivos motores para dar sentido a la dinámica de la práctica de actividades físicas de expresión y deportes artísticos?

¿Qué opciones de procesos creativos surgen durante la dinámica de la práctica de actividades físicas de expresión y deportes artísticos?

Hay dos preguntas previas a resolver para poder responder a la tercera pregunta. La tercera pregunta se corresponde con el objetivo del artículo.

\section{OBJetIVOS MOtORES: LOGRAR FORMAS MOTRICES}

Hernández Moreno (Dir.) (2000) indica el objetivo motor "reproducir modelos". Según Troya (2018), ese objetivo motor identifica a los deportes artísticos durante la competición (patinaje artístico, bmx, gimnasia artística, natación artística, half-pipe) y durante la representación de espectáculos (musicales, circo, performances, ópera).

Mateu y Bortoleto (2011) prefieren utilizar el término "formas motrices" para identificar el objetivo motor de las actividades de expresión. De acuerdo a este concepto, un doble mortal en cama elástica, los saltos con la comba, la cara de triste en teatro mimo, un 360 en surf, los golpeos en las katas de karate, las trayectorias en la polca americana, las maneras de encestar en la competición de mates de baloncesto, la figura del lobo en sombras chinescas y el equilibrio arabesque del ballet, son formas motrices.

El concepto de forma motriz abarca un amplio espectro. ¿Cómo se pueden agrupar las formas motrices? Hernández Moreno (Dir.) (2000) utiliza el criterio "tipo de formas motrices":

- Formas espaciales (p. e. las trayectorias de los participantes en un baile tradicional).

. Formas temporales (p. e. el ritmo en un ejercicio de percusión).

- Formas gestuales ( $p$. e. la facialidad y corporalidad en una representación de teatro mimo). malabares).

- Formas de dominio de objetos (p. e. la columnas con las bolas de 
Mateu y Torrents (2012) adoptan el criterio "tipo de soportes para realizar las formas motrices":

. Formas realizadas sobre sí mismo (p. e. salto mortal, una expresión facial de "asombrado").

. Formas realizadas conjuntamente a otros participantes (p. e. espiral de la muerte en patinaje artístico de parejas; representar un "abrazo" con el otro).

- Formas realizadas a elementos materiales (p. e. lanzamiento de la pelota en gimnasia rítmica; hacer "caricias" a una pelota que representa una persona).

Tabla 1 - Las formas motrices (objetivos motores) en las actividades de expresión y los deportes artísticos

\begin{tabular}{|l|l|}
\hline Tipo de formas & Soportes de las formas \\
\hline Las formas son: & Las formas se realizan: \\
- Espaciales & - Sobre sí mismo \\
- Temporales & - Junto a otros \\
- Gestuales & participantes \\
- De dominio de objetos & - Sobre elementos \\
& materiales \\
\hline
\end{tabular}

Según Ribas (2017), los objetivos motores otorgan identidad a las actividades físicas. Las actividades de expresión y los deportes artísticos son actividades físicas porque contienen objetivos motores.

La primera pregunta (¿Cuáles son los objetivos motores que identifican a las actividades físicas de expresión y a los deportes artísticos durante la dinámica de la práctica?) ha quedado resuelta. Las actividades de expresión y los deportes artísticos son actividades físicas porque sus objetivos motores durante la dinámica de la práctica son intentar obtener formas motrices.

\section{ObJetivos Externos: RefERENCIAS Estéticas Y TemÁticas}

Las realizaciones motrices de los participantes (gestuales, temporales y espaciales) carecen de significado. Parlebas (2001 y 2017) sostiene que es imprescindible asociar las intervenciones de los participantes a referencias externas para que tengan sentido.

El objetivo motor es un componente de la lógica interna, pero en contra de lo indicado en Hernández Moreno et al. (2000), las actividades de expresión y los deportes artísticos no cobran sentido con los objetivos motores. Las actividades de expresión son uno de los casos de actividades físicas señalados por Ribas (2018) en que para poder comprender la dinámica de la práctica se deben añadir objetivos externos. 
Las referencias externas se encuentran fuera de la lógica interna, en el contexto. ¿Cuáles son las referencias externas de las actividades de expresión?

Por un lado, Mateu y Bortoleto (2011), Romero (2015) y Ribas (2018) explicitan que los objetivos motores de las actividades de expresión y los deportes artísticos (las formas motrices) están asociados a referencias estéticas. Sabemos que los movimientos realizados por los participantes en una coreografía pertenecen al baile urbano colectivo, porque sus referencias estéticas son la sincronización grupal gestual y temporal, la intensidad gestual y la simetría espacial. Las realizaciones motrices de los participantes son reconocidas como formas motrices siempre y cuando se correspondan con referencias estéticas.

Los tipos de referencias estéticas se encontrarán fuera de la Praxiología motriz, por ejemplo, en Teoría del arte o en Filosofía de la Estética. Ejemplos de referencias estéticas mencionan el estilo, la armonía, el contraste, la fluidez, etc. (ver al respecto AUGUSTIN et al., 2008; LEDER et al., 2004; MORPHY; PERKINS, 2006).

Por otro lado, los objetivos motores también pueden aludir a una temática, a un asunto, es decir, a las funciones poética y referencial (MATEU; BORTOLETO, 2011; TROYA, 2018), simbólica y semiotriz (MATEU; TORRENTS, 2012; RIBAS, 2017; ROMERO, 2015). Por ejemplo, las figuras a reproducir durante la competición de gimnasia estética de grupo siempre están asociadas al desarrollo de un argumento.

Mateu y Bortoleto (2011) y Parlebas (2017) destacan que las actividades de expresión de índole temática son posibles mediante la metáfora. En las actividades de expresión, el "estar haciendo como si..." metafórico será desenmascarado cuando se logra establecer un puente entre el hacer y el significar. Las formas creadas por el participante en el juego de averiguar el nombre de la película tienen su referencia en la trama y en el título de la película.

Otra vez más hay que buscar fuera de la Praxiología motriz. Desde la Semiótica (Teoría de los signos), Eco (1992) sostiene que la metáfora deriva necesariamente hacia universos semánticos. Es decir, las realizaciones de los participantes serán reconocidas como formas motrices cuando metafóricamente representen un universo semántico. Por ejemplo, los gestos de los pequeños con los muñecos pueden entenderse cuando sus referencias son personajes, roles sociales o rutinas.

Siguiendo con Eco (1992), los universos semánticos nos remiten a una enorme variedad de campos semánticos y lexicográficos, de contenidos de enciclopedias, de contextos, de géneros literarios y cinematográficos, de figuras literarias y tropos, etc. (ver, entre otros, ALTMAN, 2000; DEL MORAL, 2009; GARRIDO, 2000). 
Tabla 2 - Referencias estéticas/temáticas (objetivos externos) en las actividades de expresión y los deportes artísticos, que pueden ser asociadas a las formas motrices (objetivos motores)

\begin{tabular}{|c|c|}
\hline $\begin{array}{l}\text { Ejemplos de referencias } \\
\text { estéticas: } \\
\text { Estilo } \\
\text { Matización, detalles } \\
\text { Perfección } \\
\text { Sincronía } \\
\text { Elegancia, refinamiento } \\
\text { Amplitud, intensidad } \\
\text { Armonía, simetría } \\
\text { Fluidez, distensión, } \\
\text { soltura } \\
\text { Ritmo, cadencia } \\
\text { Contraste }\end{array}$ & $\begin{array}{l}\text { Ejemplos de referencias temáticas (se utilizan } \\
\text { metafóricamente): } \\
\text { Temas, argumentos, historias, tramas, anécdotas, } \\
\text { situaciones, noticias, cuentos } \\
\text { Emociones, sentimientos, pasiones, afectividad } \\
\text { Objetos, lugares, corporalidad, mente, personas, } \\
\text { culturas, grupos } \\
\text { Rutinas, hábitos, costumbres, normas, roles, reglas } \\
\text { Dilemas, valores (personales, culturales, morales), } \\
\text { moralejas } \\
\text { Imágenes, pensamientos, sueños, fantasías } \\
\text { Chistes, bromas, engaños, caricaturización, } \\
\text { exageraciones } \\
\text { Conceptos, ideas, creencias, verdades, opiniones, } \\
\text { conocimientos } \\
\text { Actitudes, estados de ánimo, características de la } \\
\text { persona } \\
\text { Expresiones, emblemas, símbolos, señales, códigos, } \\
\text { fotografías }\end{array}$ \\
\hline
\end{tabular}

La respuesta a la segunda pregunta es: no, no son suficientes los objetivos motores para dar sentido a la dinámica de la práctica de las actividades físicas de expresión y los deportes artísticos; los objetivos motores siempre están vinculados a objetivos externos. Luego, en las actividades de expresión, más propiamente habría que hablar de "objetivo de la actividad".

En síntesis, los objetivos de las actividades de expresión y los deportes artísticos son: intentar obtener formas motrices asociadas a referencias estéticas/temáticas.

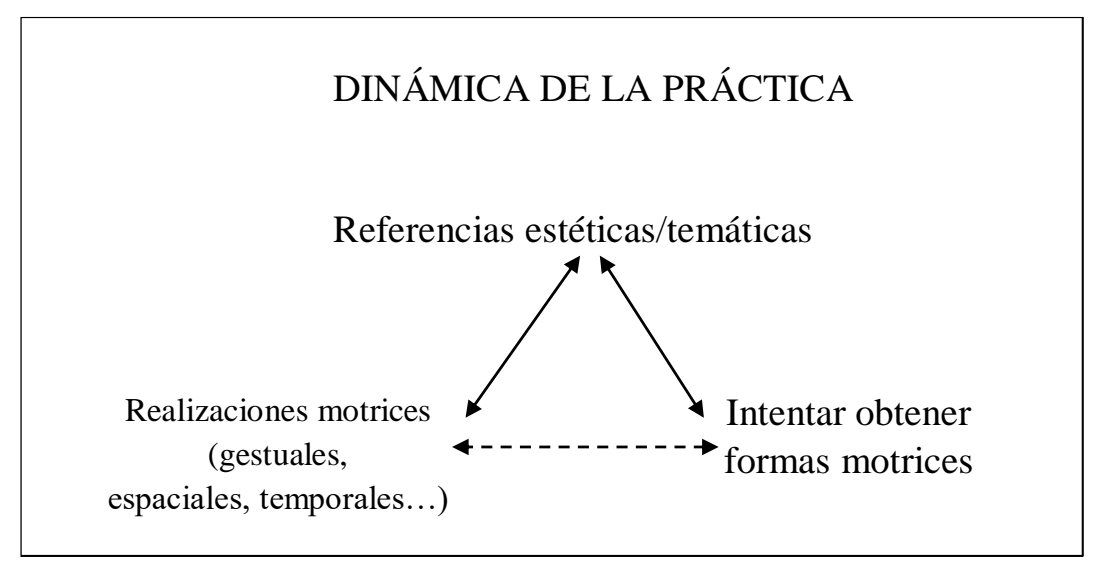

Figura 1 - Las formas motrices tienen sentido y se entienden cuando las realizaciones motrices de los participantes se asocian a referencias externas. Los objetivos de las actividades de expresión aquí se muestran en formato de signo motor expresivo. Fonte: Ribas (2017) (acorde con Eco (1988)).

Compilando hasta este punto, las actividades físicas de expresión y 
deportes artísticos pueden explicarse por los tipos de formas motrices (espaciales, gestuales, temporales) realizadas por los participantes utilizando diferentes soportes (sobre sí mismo, junto a otros, sobre objetos), formas motrices que siempre están vinculadas a referencias estéticas (cadencia, amplitud, contraste, etc.) o a referencias temáticas (ideas, sentimientos, argumentos, etc.).

\section{Opciones Creativas en las Actividades de Expresión}

Pasamos a la última pregunta. ¿Qué opciones de procesos creativos surgen durante la dinámica de la práctica de actividades físicas de expresión y deportes artísticos?

Mateu y Torrents (2012) mencionan dos procesos creativos en Expresión corporal: la improvisación y la elaboración; Castañer et al. (2009) y Ribas (2018) señalan los procesos de improvisación y de variación; Romero, (2015) y Troya (2018) también nombran la improvisación como opción creativa.

¿Qué diferencias existen entre unos y otros procesos de creatividad motriz? ¿Y qué diferencias tienen con respecto a la reproducción?

Como paso previo, sugerimos indicadores de los cuatro procesos:

. Imitar: reproducir, copiar, repetir, buscar similitudes, asemejar, igualar.

. Variar: diferenciar, alterar, modificar, buscar alternativas, cambiar, transformar, derivar, diversificar.

. Componer: elaborar, construir, escoger, relacionar, añadir, acoplar, combinar, ajustar, reorganizar.

. Improvisar: inventar, imaginar, idear, ser ingenioso, descubrir, suponer, originar, surgir, innovar, indagar.

(El profesorado puede utilizar estos términos en el momento de diseñar tareas expresivas y en el momento de dirigirse a sus alumnos.)

Los participantes se desenvuelven en la dinámica de la práctica intentando alcanzar el objetivo de la actividad (HERNÁNDEZ MORENO (Dir.), 2000). Recordemos. Los objetivos de las actividades de expresión y los deportes artísticos son: intentar obtener formas motrices asociadas a referencias estéticas/temáticas, de lo cual resulta que:

. En el proceso de imitar los participantes tratan de repetir formas motrices manteniendo idénticas referencias estéticas o temáticas. 
. Inversamente, durante el proceso de improvisación los participantes inventan formas motrices cambiantes mientras descubren temas o indagan referencias estéticas.

. En el proceso de composición los participantes van escogiendo formas motrices y ajustando las referencias estéticas/temáticas, para finalizar en una combinación repetible.

. A la inversa, en el proceso de variación los participantes parten de un modelo y, progresivamente, modificarán las formas motrices y alterarán las referencias estéticas/temáticas.

¿Cuál es el grado de creatividad de los cuatro procesos? ¿Cómo funciona la creatividad en los cuatro procesos creativos? Troya (2018, p. 33) indica que "En cualquier práctica motriz en la que haya incertidumbre, se puede ser creativo a la hora de ofrecer respuestas novedosas y válidas al problema motor en cuestión".

Se puede diferenciar el grado de creatividad de los procesos, poniendo en relación el grado de codificación (reglamentación) de la actividad (MATEU; BORTOLETO, 2011) y, debido a ello, el consecuente grado de incertidumbre que tienen los participantes durante la dinámica de la práctica (RIBAS, 2018):

. La imitación necesita un grado de reglamentación alto y, debido a ello, la incertidumbre que tiene el participante durante la práctica es mínima. La creatividad es escasa y está predeterminada: los participantes se limitan a averiguar si lo que están intentando imitar es igual al modelo o patrón motor previsto y si están manteniendo las referencias (estéticas/temáticas). Es por eso que durante la imitación el profesorado suele insistir en la repetición y en la precisión de los detalles. La creatividad determinista se da durante la reproducción de bailes tradicionales (p. e. una sevillana), durante la competición deportiva (p. e. baile deportivo) o durante un espectáculo (p. e. magia).

- La improvisación requiere la ausencia de código y de reglas, es decir, la incertidumbre que tienen los participantes durante la práctica es alta. La creatividad no está determinada: potencialmente la creatividad podría llegar a ser máxima, por ejemplo, durante el juego de continuar la historia (mediante mímicas), durante el juego del espejo, durante los juegos con roles sociales ( $p$. e. médicos y enfermeros) y durante la danza-contacto. En la creatividad indeterminista de la improvisación, el participante se va deslizando entre sus propios puntos de vista, mientras el profesorado suele incentivar la no repetición, la constante exploración y la búsqueda, precisamente para evitar que los alumnos desemboquen en una rutina.

- La creatividad semi-determinista surge al intentar variar o al intentar componer. En ambos procesos hay una actividad reglamentada como punto de 
partida (p. e. intentar buscar variantes de la coreografía de un musical) o una actividad reglamentada como punto de llegada ( $p$. e. intentar componer las secuencias para una competición de skate en half pipe). En consecuencia, el grado de incertidumbre que tienen los participantes durante la actividad es medio: los participantes van proponiendo un abanico de posibilidades, que van aumentando en las variaciones, o posibilidades que se van reduciendo en el proceso de componer.

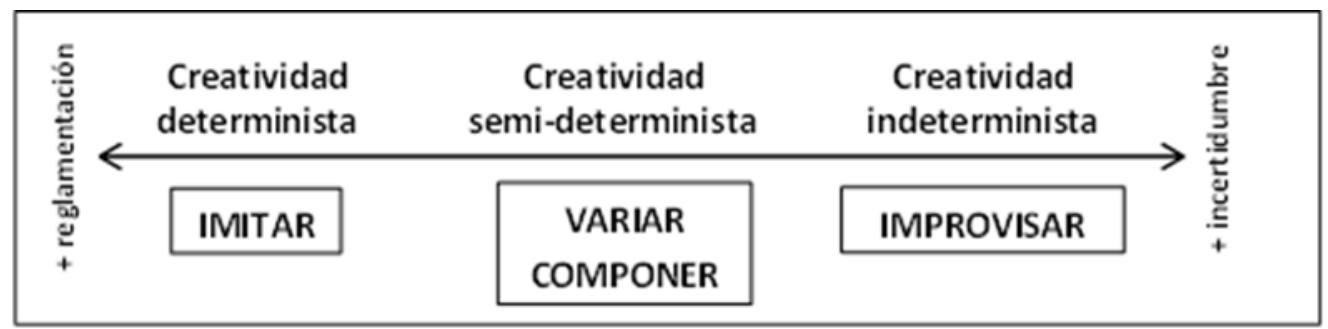

Figura 2 - Diferentes grados de creatividad de los cuatro procesos, en las actividades de expresión y deportes artísticos. La reglamentación de la actividad se muestra inversa a la incertidumbre generada en los participantes.

\section{CONSIDERACIONES FINALES}

Se ha planteado este artículo con el objetivo de determinar las opciones de creatividad durante la práctica de las actividades físicas de expresión y los deportes artísticos desde la Spórtica.

La Spórtica analiza la dinámica de la práctica, es decir el momento en que los participantes están practicando. Para sus análisis, la Spórtica utiliza criterios como el tipo de objetivos motores, el tipo de objetivos externos y el tipo de incertidumbre. En este artículo se ha argumentado que:

- Los objetivos motores "obtener formas motrices" identifican a las actividades de expresión y a los deportes artísticos como pertenecientes al universo de las actividades físicas y deportivas. Las formas motrices (espaciales, gestuales, temporales) se realizan en diversos soportes (sobre sí mismo, junto a otros, sobre los objetos).

- Los objetivos motores requieren objetivos externos para que las actividades de expresión y los deportes artísticos tengan sentido, concretamente las referencias estéticas (cadencia, amplitud, contraste, etc.) y las referencias temáticas (ideas, sentimientos, argumentos, etc.). Los objetivos de las actividades de expresión y los deportes artísticos son "intentar obtener formas motrices asociadas a referencias estéticas/temáticas".

. Imitar, improvisar, variar y componer son cuatro procesos creativos. Durante la imitación los participantes reproducen formas motrices con idénticas 
referencias estéticas/temáticas. Durante la improvisación los participantes inventan formas motrices efímeras vinculadas a referencias estéticas/temáticas cambiantes. Durante la variación los participantes modifican formas motrices y alteran las referencias a partir de un modelo originario. Durante la composición los participantes escogen formas motrices y eligen sus correspondientes referencias estéticas/temáticas.

- Se descubren tres grados de creatividad durante la dinámica de la práctica: mínimo durante la imitación, intermedio durante la variación y la composición y potencialmente máximo durante la improvisación. La razón de las diferencias creativas estriba en los diferentes grados de reglamentación de la actividad que se requiere para cada proceso y la consecuente incertidumbre que producen en el participante durante la práctica.

En conclusión, y desde la Spórtica, el profesorado de Educación física tiene a su disposición cuatro procesos creativos agrupados en tres grados de creatividad.

Hay temas relacionados con la creatividad en las actividades de expresión no tratados en el presente artículo:

- La posible creatividad procedente de otras opciones de incertidumbre. Por ejemplo, la incertidumbre generada en los participantes practicando en un medio físico fluctuante o desconocido (por ejemplo, las olas y el viento en kitesurf freestyle). 0 , por ejemplo, la incertidumbre generada en los participantes cuando intentan formas motrices y referencias estéticas/temáticas difíciles de alcanzar.

- La posible creatividad procedente de la práctica de diversidad de opciones: variedad de formas motrices, realizadas sobre variedad de soportes, asociados a variedad de referencias estéticas y variedad de referencias temáticas.

\section{REFERENCIAS}

ALTMAN, Rick. Los géneros cinematográficos. Barcelona: Paidós, 2000.

AUGUSTIN, Dorothee; LEDER, Helmut; HUTZLER, Florian; CARBON, Claus Christian. Style follows content. On the microgenesis of art perception. Acta Psychologica, v. 128, n. 1, p. 127-138, 01 may. 2008. Disponible en:

https://doi.org/10.1016/j.actpsy.2007.11.006 Acceso: 22 feb. 2020.

CASTAÑER, Marta; TORRENTS, Carla; DINUŠOVÁ, María; ANGUERA, Teresa.

Instrumentos de observación ad hoc para el análisis de las acciones motrices en Danza Contemporánea, Expresión Corporal y Danza Contact-Improvisación. Apunts Educación Física y Deportes, v. 95, n. 1, p. 14-23, 01 ene. 2009. Disponible en: 
https://www.revista-apunts.com/apunts/articulos//95/es/095 014-023 es.pdf Acceso: 06 ene. 2020.

DEL MORAL, Rafael. Diccionario ideológico. Atlas léxico de la lengua española. Barcelona: Herder, 2009.

ECO, Umberto. Signo. Barcelona: Labor, 1988.

ECO, Umberto. Los límites de la interpretación. Barcelona: Lumen, 1992.

GARRIDO, Miguel Ángel. Nueva introducción a la teoría de la literatura. Madrid: Síntesis, 2000.

HERNÁNDEZ MORENO, José. (Dir.). La iniciación a los deportes desde su estructura y dinámica. Aplicación a la Educación Física Escolar y al Entrenamiento Deportivo.

Barcelona: INDE, 2000.

HERNÁNDEZ, José; CASTRO, Ulises; CRUZ, Heriberto; GIL, Guillermo; HERNÁNDEZ, Luz Marina; QUIROGA, Miriam; RODRÍGUEZ, Juan Pedro. ¿Taxonomía de las actividades o de las situaciones motrices? Apunts, Educación Física y Deportes, v. 60, n. 2, p. 95-99, 01 abr. 2000. Disponible en: https://www.revistaapunts.com/apunts/articulos//60/es/60 095 100 ES.pdf Acceso: 07 dic. 2019.

LEDER, Helmut, BELKE, Benno; OEBERST, Andries; AUGUSTIN, Dorothee. A model of aesthetic appreciation and a esthetic judgments. British Journal of Psychology, v. 95, p. 489-508, 2004. Disponible en:

http://citeseerx.ist.psu.edu/viewdoc/download?doi=10.1.1.395.5700\&rep=rep1\&type=pd f Acceso: 19 ene. 2020.

MATEU, Mercè; BORTOLETO, Marco Antonio. La lógica interna y los dominios de acción motriz de las situaciones motrices de expresión (SME). Emancipaçao, v. 11, n. 1, p. 129142, ene/abr 2011. Disponible en:

https://www.revistas2.uepg.br/index.php/emancipacao/article/view/2463/2357 Acceso: 23 nov. 2019.

MATEU, Mercè; TORRENTS, Carlota. La lógica interna de las actividades físicas artísticoexpresivas. Tándem. Didáctica de la Educación Física, v. 39, n. 2, 48-61, 01 abr. 2012. Disponible en: https://www.grao.com/es/producto/logica-interna-de-las-actividadesfisicas-artistico-expresivas Acceso: 23 nov. 2019.

MORPHY, Howard; PERKINS, Morgan (Eds.). The Anthropology of Art: A Reader. Malden, MA: Blackwell Publishing, 2006.

PARLEBAS, Pierre. Juegos, deporte y sociedad: Léxico de Praxiología motriz. Barcelona: Paidotribo, 2001.

PARLEBAS, Pierre. La aventura praxiológica: Ciencia, Acción y Educación Física. Málaga: IAD, 2017.

RIBAS, Juan Pedro. Acción, signo motor y Semiotricidad. Acción motriz, v. 18, n.1, p. 4762, 01 ene. 2017. Disponible en:

http://www.accionmotriz.com/documentos/revistas/articulos/18 5.pdf. Acceso: 23 nov. 2019.

RIBAS, Juan Pedro. Spórtica y clasificación del deporte y las actividades físicas. Lecturas: Educación Física y Deportes, v. 23, n. 246, p. 123-139, 23 nov. 2019. Disponible en: 
https://www.efdeportes.com/efdeportes/index.php/EFDeportes/article/view/481/431. Acceso: 23 nov. 2019.

ROMERO, María Rosario. Expresión corporal en Educación Física. Zaragoza: Prensas universitarias de Zaragoza, 2015.

TROYA, Yanira. La expresión corporal en la educación física escolar. Una propuesta de estructuración de los contenidos expresivos. Acción motriz, v. 20, n. 1, p. 27-36, 01 ene. 2018. Disponible en:

http://www.accionmotriz.com/documentos/revistas/articulos/20 3.pdf Acceso: 20 nov. 2019. 